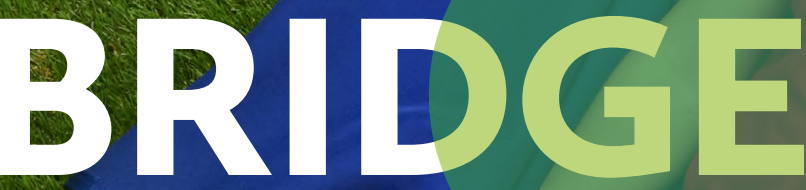

September 2019

\title{
Resilience Edition
}

Cognitive processes mediate the post-traumatic stress trajectory in adolescents

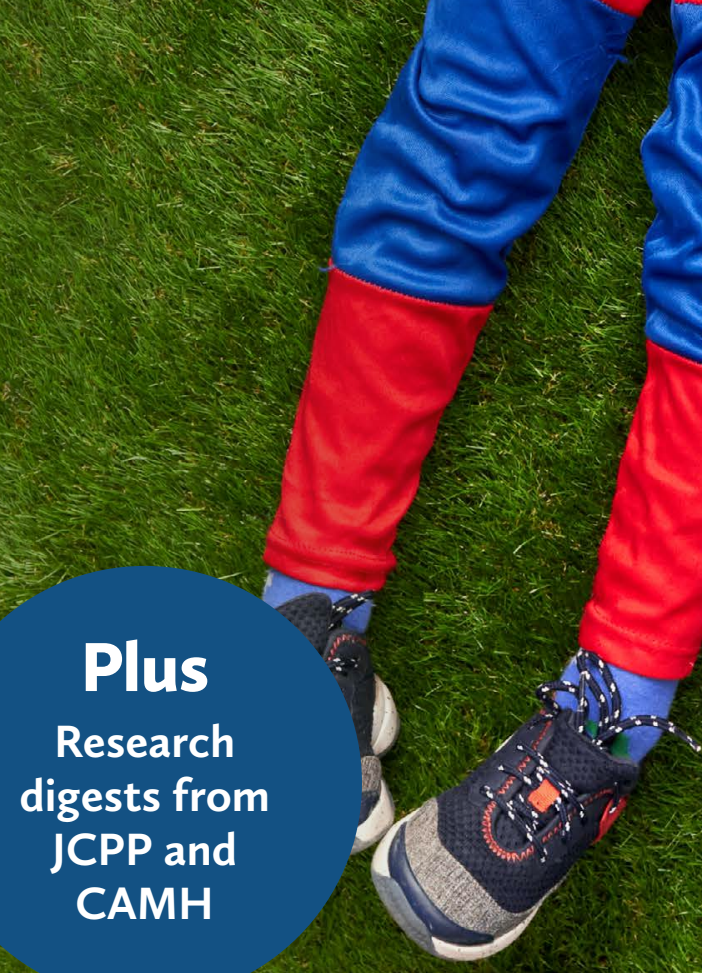




\title{
Editorial
}

\author{
The Bridge Editor, Dr Juliette Kennedy
}

Welcome to The Bridge. In this edition we bring together a number of papers that broadly discuss the theme of resilience and developing resilience through therapy. Resilience can be described as having the capacity to recover quickly from difficulties or to be able to "spring back into shape". It may explain why some young people are more vulnerable and some young people more resilient to similar stressors or adverse childhood experiences.

Dunning et al. comment that Mindfulness-based interventions are generally popular at present and they also raise the suggestion that enthusiasm may have moved ahead of the evidence base. They have therefore undertaken a systematic literature search to explore the current evidence base for Mindfulness based interventions in young people.

Bennet et al. highlight that the demand for treatment in CAMHS services outweighs supply and that waiting lists for CAMHS services can be long. They look at the efficacy of using 'low intensity' treatments, such as self-help materials, as a way of increasing young people's access to evidencebased psychological treatments. Results look positive, but they highlight that the challenge is knowing what type of self-help would be better suited to which patients. Resilience may be a factor in this.

Meiser-Stedman et al. report their prospective longitudinal study exploring how cognitive processes may play an important role in the onset and maintenance of PTSD symptoms in some young people exposed to trauma. Participants were categorized as being on a resilient, recovery or persistent trajectory. This resonates with clinical experience of how different young people respond differently to similar traumatic events.

Gillespie et al. compare the 16-week and 24-week DBT programmes delivered by CAMHS teams in Ireland to determine whether a longer DBT-A programme would yield improved outcomes for participants. The cornerstones of DBT therapy: Mindfulness, interpersonal effectiveness, distress tolerance and emotional regulation, all aim to build resilience over time. The question of how much time in therapy is needed clearly has significant service delivery and cost implications.

Martin et al. highlight that children with ADHD often present with other mental health symptoms (anxiety, depression, loneliness) but that interestingly, young people with ADHD may also have a positive self-perceptual bias (e.g. an overestimation of their own abilities) and that this may hinder the accurate assessment of other mental health problems, so symptoms of anxiety, depression, and loneliness may be underestimated in these young people. This was surprising to read but on reflection, did not feel unfamiliar in my experience of clinical practice.

I do hope you find this edition helpful and interesting.

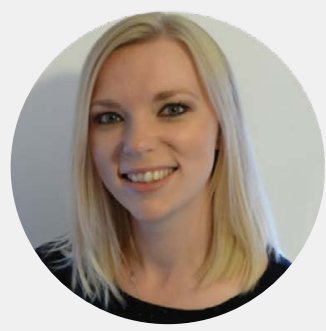

Research highlights in this edition are prepared by Dr Jessica K Edwards. Jessica is a freelance editor and science writer, and started writing for 'The Bridge' in December 2017. 
By Jessica K. Edwards

A new study has shown that cognitive processes shape the early reactions of children and adolescents to traumatic stressors, and mediate the transition to persistent and clinically significant post-traumatic stress symptoms (PTSS). Meiser-Stedman and colleagues conducted a prospective, longitudinal study of $>200$ youths aged 8-17 years who had attended hospital after experiencing a single trauma. They made assessments at 2-4 weeks and 2 months post-trauma, using structured interviews and self-report questionnaires to categorize each patient as being on a resilient, recovery or persistent PTSS trajectory.

The data showed that cognitive processes at the 2-4 week assessment were the most powerful predictors of the onset and maintenance of PTSS. The onset of acute, clinically significant PTSS was associated with perceived threat, data-driven processing ${ }^{1}$ and pain, while the maintenance or persistence of PTSS at the 2-month follow-up was associated with more poorly elaborated, sensory-based trauma memories, persistent dissociation, trauma-related rumination and negative appraisals of the trauma.

Overall, the researchers found that very early reactions to trauma in youths may be conceived of as a common reaction to the trauma and that in many cases, such reactions will diminish over the coming weeks. The presence of negative appraisals of the trauma and its sequelae, however, disrupts these recovery processes. Given the novelty of these findings, the researchers concede that more research into the mechanisms of PTSS is needed before any strong recommendations regarding clinical interventions are made. They do suggest, however, that their data might support targeting negative appraisals in the psychological prevention and treatment of post-traumatic stress disorder. In addition, they propose that there is a need to better recognise and address ruminative thinking styles (rather than only avoidance) in youths affected by trauma.

\section{References}

Meiser-Stedman, R., McKinnon, A., Dixon, C., Boyle, A., Smith, P. and Dalgleish, T. et al. (2019), A core role for cognitive processes in the acute onset and maintenance of post-traumatic stress in children and adolescents. J. Child Psychol. Psychiatr. 60:875-884. doi: 10.1111/jcpp.13054.

\section{Further reading}

'Ehlers, A. et al. (2000), A cognitive model of posttraumatic stress disorder. Behav. Res. Ther. 38:319-345. doi: 10.1016/So005-7967(99)oo123-0.

\section{Glossary}

Data-driven processing: A sensory analysis that focuses on incoming sensory data, which is then transmitted to the brain and processed to form a visual experience. This form of processing requires no previous knowledge or learning; as such, it is also known as bottom-up processing, as opposed to top-down (conceptual) processing. In the context of trauma, data-driven processing refers to the processing of the sensory impression of the traumatic situation and the perceptual characteristics, rather than the meaning of the event. Here, data-driven processing is thought to result in a poorly elaborated, perceptually encoded memory trace, whereas top-down processing will result in contextualized memory representations. ${ }^{1}$

Dissociation: A feeling of being disconnected from a sensory experience, such as a traumatic event, or from one's self or personal history.

Rumination: A tendency to repetitively think about the causes, situational factors and consequences of a negative emotional experience or trauma. 


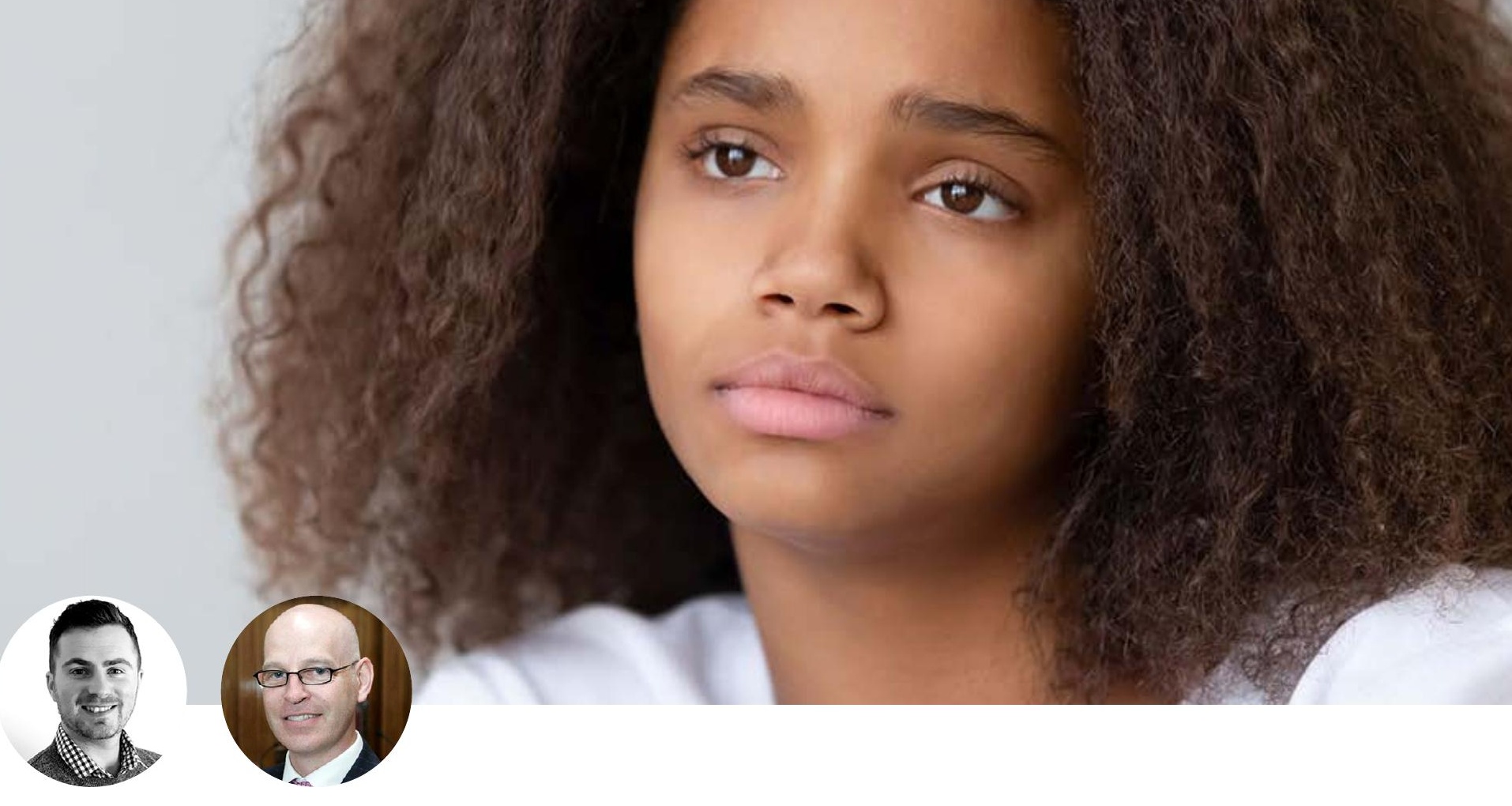

\section{Dialectical behaviour therapy for} adolescents: a comparison of 16-week and 24-week programmes delivered in a public community setting

By Conall Gillespie and Daniel Flynn

Conall Gillespie is a Trainee Clinical Psychologist in the Health Service Executive (HSE), Ireland. He is currently completing the Doctor of Clinical Psychology in University College Cork, Ireland.

Daniel Flynn is a Clinical Psychologist and Principal Psychology Manager co-ordinating Adult and Child/ Adolescent Mental Health Services in the HSE across counties Cork and Kerry, Ireland and is Adjunct Professor at the School of Applied Psychology University College Cork, Ireland. His clinical interests are in working with individuals who struggle to regulate emotions and engage in high risk self harm behaviours. He is also interested in the impact of these behaviours on families.

This article is a summary of the paper published in CAMH - Gillespie C, Joyce M, Flynn D, Corcoran P. (2019). Dialectical behaviour therapy for adolescents: a comparison of 16-week and 24-week programmes delivered in a public community setting. Child and Adolescent Mental Health. doi: 10.1111/camh.12325
Self-harm among adolescents is a growing problem. Registries of hospital treated self-harm in the U.K. and Ireland both report the highest rates in younger age groups. Clinicians in Child and Adolescent Mental Health Services (CAMHS) also report an increase in the number of adolescents presenting to their services with self-harm and/or suicidal behaviour in recent years. Despite the large number of adolescents and their families requiring support, interventions for this group of individuals are still quite limited. One intervention with an emerging evidence base for treating emotionally dysregulated adolescents with self-harm/ suicidal behaviours is dialectical behaviour therapy for adolescents (DBT-A).

Clinicians in CAMHS across Ireland have sought training in DBT-A to treat the increasing number of adolescents presenting to their services with self-harm and/or suicidal behaviour. With a growing national interest in DBT provision in community services, the National DBT Project Ireland was established in 2013 to coordinate training and implementation of DBT (in both adult and child/adolescent services) in the Irish 

all outcome measures from pre- to post-intervention. The findings however did suggest that 24-week DBT-A may have additional benefits in comparison to 16 -week DBT-A in terms of further reductions in suicidal ideation and depression. Due to the nature of our study, it was not possible to measure outcomes for the two groups at both 16 weeks and 24 weeks. As a result, it is not possible to determine whether the more positive results in the 24 -week group are due to the extra eight weeks of intervention or due to the passage of time.

The differences in drop-out rates for the two programmes must also be considered in the context of the results as it may have important feasibility implications. Given the high level of risk associated with self-harm and emotional dysregulation, the shorter 16-week programme may be preferable in order to retain more participants. Despite the drop-out rate being higher for the 24-week group, it is important to mention that this figure is comparable to other DBT-A studies.

It is possible that by having a longer programme, additional benefits may be observed for individuals participating in DBT-A. However, in order to provide an extended treatment, there is an increased resource and time commitment required by DBT therapists. Therefore, future research will need to focus on an economic evaluation to compare the economic effectiveness of the 16-week and 24-week DBT-A programmes. This will provide important information in determining optimal levels of clinical input to yield positive outcomes for clients. Future research might also consider long term follow-up to identify if gains made at post-intervention are maintained for participants who complete 16-week and 24-week DBT-A.

\section{Key points:}

\section{Clinical practice:}

- Significant improvements on constructs such as self-harm behaviour and suicidal ideation were reported in both 16 -week and 24-week DBT-A programmes.

\section{Service development/delivery:}

- 24-week DBT-A programmes may be more effective in reducing suicidal ideation and depression.

\section{Gaps and recommendations for further scientific enquiry:}

- Further research is required to identify optimal treatment duration of DBT-A.

- Longitudinal studies are required to explore treatment outcomes for 16 versus 24 -week interventions in the longer term. 


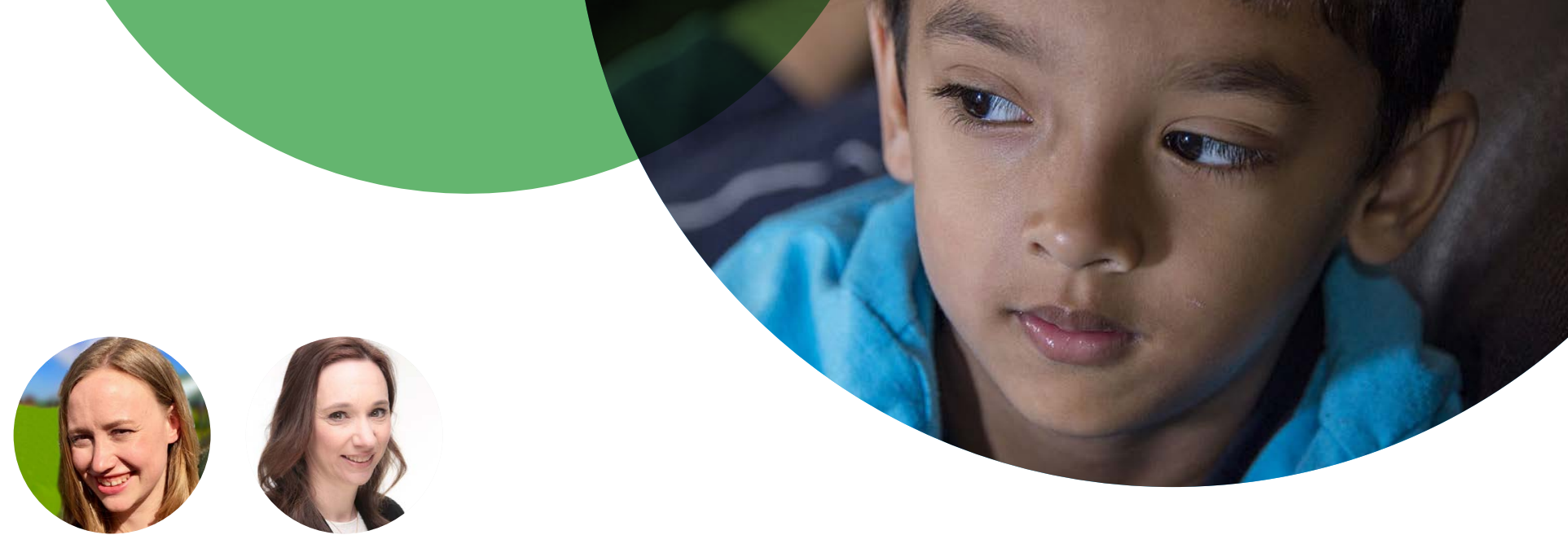

\title{
Help yourself (but get some support): meta-analysis of self-help interventions for children
}

\author{
By Sophie D. Bennett \& Roz Shafran
}

\section{Sophie Bennett is a Senior Research Fellow at the UCL Great Ormond Street Institute of Child Health and Honorary Clinical Psychologist at Great Ormond Street Hospital for Children. She is involved in a number of studies investigating the use of low-intensity or telephone treatments to increase access to psychological therapies in children and young people and particularly in those with chronic physical illnesses.}

\section{Roz Shafran is Professor of Translational Psychology at the UCL Great Ormond Street Institute of Child Health and Honorary Consultant Clinical Psychologist at Great Ormond Street Hospital for Children. She leads the Psychological Medicine Research Team which focuses on evaluating the psychological treatments that have been shown to work in young people with mental health disorders in those who also have physical health conditions.}

This article is a summary of the paper published in JCPP - Bennett S. D, Cuijpers P, Ebert D. D, Smith M. M, Coughtrey A. E, Heyman I, Manzotti G. (2019). Practitioner Review: Unguided and guided self-help interventions for common mental health disorders in children and adolescents: a systematic review and metaanalysis. Journal of Child Psychology and Psychiatry. doi: 10.1111/jcpp.13010

Mental health disorders affect $12.8 \%$ of children and young people in the UK. We know that poor mental health in childhood is associated with poorer quality of life, increased likelihood of having a mental health disorder in adulthood and poorer occupational and social outcomes. We also know that there are numerous effective interventions for mental health problems, with over 750 treatment protocols for evidence-based psychological treatments in children. However, demand for treatment outstrips supply. Unfortunately, many children with mental health problems do not receive support and there are often extensive waiting lists for Child and Adolescent Mental Health Services (CAMHS), which are increasingly overstretched. Recent national data indicated that $36 \%$ of referrals to CAMHS are closed before any treatment is received and for those accepted, $30 \%$ of children were assessed within four weeks of referral. Half of the 11,482 children needing treatment following this assessment waited more than 18 weeks to receive it.

One way of increasing access to evidence-based psychological treatments is through 'low-intensity' interventions, such as self-help delivered through books or online, with or without guidance from a therapist or trained supporter. These interventions are relatively inexpensive as they do not require extensively trained therapists and are more accessible as they can be delivered remotely so families do not have to travel to attend appointments, reducing time off work or school. Such low-intensity interventions are a fundamental part of a 'stepped care' approach to the delivery of interventions, in which the least resource-intensive intervention that is effective for the presenting difficulty is offered first, before treatments that are more intensive. Low intensity interventions can be offered whilst children are on waiting lists for more intensive treatments. Stepped care therefore allows for more efficient services and prevents 'over-treating' those where a brief, low-intensity intervention would be effective and sufficient. 
Such a model has been used successfully for several years within the adult Improving Access to Psychological Therapies (IAPT) Programme. Within CAMHS, the use of low-intensity interventions has been implemented by a new workforce of Child Wellbeing Practitioners (CWPs). This new workforce was a response to the Five Year Forward View for Mental Health and was outlined in the associated implementation plan. Specifically, the Department of Health and Social Care committed to training 1700 new staff in evidence-based treatments in order to offer these to 70,000 more children and young people annually by 2020 . More recently, the government has announced the implementation of mental health support teams in schools, which will support children and young people with mild to moderate mental health issues, in part through training 'education mental health practitioners' to deliver low-intensity interventions such as guided self-help.

Whilst several Randomised Controlled Trials and metaanalyses have demonstrated that self-help interventions are effective, it is important to know how they should be implemented in practice in order to maximise their benefit. If they are likely to be ineffective in some circumstances, this is important to know, as offering an ineffective treatment could further delay access to effective treatment and some children may be reluctant to access further treatment if they have a difficult experience with self-help. Services need to know who self-help interventions should be offered to, under what circumstances and how they should be offered. For example, regarding who they should be offered to, should we only offer it to teenagers or does it work for younger children too? Regarding under what circumstances, does it make a difference if a parent is present to provide support or not? Regarding how they are delivered, does it matter if there is guidance and if so, who the guidance is delivered by or how it is delivered? Does the number of sessions affect outcome? Are online interventions better than books? Finally, when considering their use within a stepped-care model, it is important to know how they compare to higher intensity, more traditional face-toface interventions.
This paper assesses the extent to which these various intervention and patient factors are associated with the outcome of self-help therapies in children and young people. We comprehensively searched for all of the trials of self-help interventions for common mental health problems (anxiety, depression and behavioural difficulties) in children and young people. Fifty studies met our inclusion criteria. Overall, when the results of the 50 studies were combined, we found that self-help interventions demonstrated similar efficacy to standard face-to-face treatments. Interventions delivered with some guidance may be more effective than those delivered without guidance (i.e. pure self-help). Most moderating variables (such as severity or type of guidance) did not have any effect on outcome, however this needs further research with well-conducted studies. In particular, it would be beneficial to compare low-intensity interventions with higher intensity interventions and to directly compare different types of self-help. The results to date suggest that self-help with guidance is effective for common mental health disorders in children and young people and is a viable method of ensuring those who need treatment are able to access it.

\section{Key points:}

- Using 'low intensity' treatments, such as self-help materials, may be one way to increase access to evidence-based psychological treatments.

- Our research suggests that they may be as effective as standard 'face to face' treatments when offered with guidance from a therapist or supporter.

-We need more research that compares different types of self-help to know what types of selfhelp would be better suited to which patients.

Author correspondence: Sophie D. Bennett, UCL Great Ormond Street Institute of Child Health, 30 Guildford Street, London, WC1N IEH, UK; Email: sophie.bennett.10@ucl.ac.uk 


\title{
entions improve
} depression and anxiety outcomes in youths

\author{
By Jessica K. Edwards
}

Mindfulness describes a natural mental state in which an individual's attention is directed towards the present moment, with an attitude of accepting one's feelings, thoughts, sensations and the surrounding environment, non-judgementally, just as it is. High levels of mindfulness practice have been associated with better functioning for various psychological and physical health outcomes;' so, augmenting mindfulness practice via mindfulness-based interventions (MBIs) has been hypothesized to have downstream effects on more distal outcomes such as improved behaviour or reduced symptoms of psychopathology. ${ }^{2}$

In 2019, Darren Dunning and colleagues compiled a Research Review for the Journal of Child Psychology and Psychiatry in which they evaluated, for the first time, the efficacy of MBls on cognition and mental health in adolescents $<18$ years-of-age. They conducted a meta-analysis of 33 randomized controlled trials (RCTs) that together included $>3,600$ children, and found that across all RCTs, there was a significant positive effect of MBls compared to control on the following outcome categories: Mindfulness, Executive Functioning, Attention, Depression, Anxiety/Stress and Negative Behaviours. Of note, however, only small-tomoderate effect sizes were found (Cohen's d: 0.16-0.30) across these categories. When considering only the RCTs that included an active control group $(n=17)$, the significant benefits were restricted to outcomes of Mindfulness, Depression and Anxiety/Stress.

This finding suggests that studies with passive control groups may wrongly inflate the effects of an $\mathrm{MBI}$ on some outcome domains.

The researchers conclude that while the superiority of MBls over active comparison conditions for these outcomes is reassuring, more work is needed to directly compare MBls with psychological and psychosocial interventions that aim to reduce mental health problems or improve wellbeing. Overall, Dunning et al. advocate the use of MBls to improve mental health in young people but reinforce that further RCTs are needed to evaluate the robustness of MBIs in youths, with a focus on the mechanisms of action.

\section{References}

Dunning, D.L., Griffiths, K., Kuyken, W., Crane, C., Foulkes, L., Parker, J. and Dalggleish, T. (2019), Research Review: The effects of mindfulness-based interventions on cognition and mental health in children and adolescents - a meta-analysis of randomized controlled trials. J. Child Psychol. Psychiatr. 60: 244-258. doi: $10.1111 /$ jcpp.12980.

\section{Further reading}

${ }^{1}$ Baer, B. et al. (2006), Using self-report assessment methods to explore facets of mindfulness. Assessment. 13:27-45. doi: 10.1177/1073191105283504.

${ }^{2}$ Khoury, B. et al. (2013). Mindfulness based therapy: A comprehensive meta-analysis. Clin. Psychol. Rev. 33, 763-771. doi: 10.1016/j.cpr.2013.05.005.

\section{Glossary}

Mindfulness-based interventions (MBIs): MBIs aim to train individuals to cultivate and incorporate mindfulness into daily life. Education is provided in formal and informal mindfulness meditation practices that train the attentional control component and the non-judgemental attitudinal aspects of mindfulness. Formal practices include sitting meditation, mindful movements and mindful explorations of bodily sensations (known as the "body scan"). Informal practices aim to integrate mindfulness into everyday activities. Classes are held in a group setting, but daily home practice is also required. 


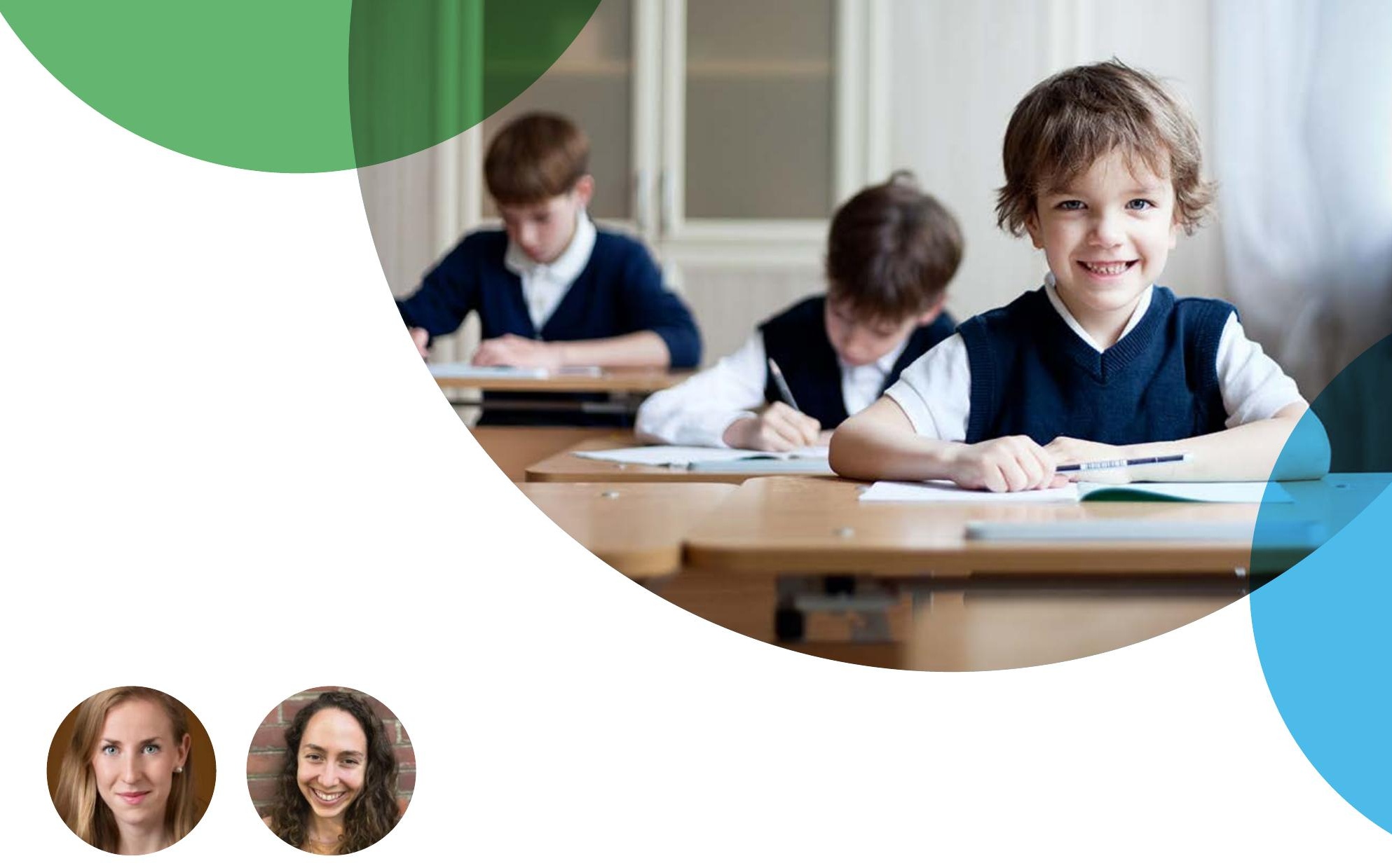

\section{Self-Perceptual Bias and Internalizing Symptoms: Implications for ADHD}

By Caroline Martin and Virginia Peisch

Caroline Martin is a fourth-year graduate student in the Clinical Psychology Ph.D. program at the University of Vermont. Working under the mentorship of Dr. Betsy Hoza, Caroline's research examines the assessment and treatment of Attention-Deficit/Hyperactivity Disorder (ADHD) from a developmental psychopathology perspective, with a specific focus on the early childhood years. In her clinical work, Caroline focuses on the treatment of ADHD, disruptive behaviors, and internalizing problems among children and adolescents.

Virginia Peisch is currently a fifth-year graduate student in the combined Developmental and Clinical Ph.D. program at the University of Vermont. As a member of the Risk and Resilience Lab at the University of Vermont, Virginia is particularly interested in studying coping processes in developing children and how coping relates to adaptive and maladaptive outcomes. In her clinical work, Virginia focuses primarily on internalizing problems in middle childhood as well as on parenting interventions.
This article is a summary of the paper published in JCPP - Martin C. P, Peisch V, Shoulberg E. K, Kaiser N, Hoza B. (2019). Does a social self-perceptual bias mask internalizing symptoms in children with attention-deficit/hyperactivity disorder?. J.Child Phsychol. Psychiatr. . doi: 10.1111/

jcpp.13024

Attention-Deficit/Hyperactivity Disorder (ADHD) is a common childhood neurodevelopmental disorder, affecting $5-7 \%$ of school-aged children. ${ }^{1}$ Given that many children with ADHD experience difficulty paying attention and managing impulsive behaviors, it is not surprising that children with ADHD often struggle with basic tasks, such as schoolwork, daily routines, and social interactions. What's more, children with ADHD often experience one or more coexisting mental health problems, such as anxiety and depression, which can further limit their overall wellbeing and daily functioning. ${ }^{2,3}$

In light of the challenges children with ADHD often face, it is important to identify ADHD and coexisting mental health problems in order to provide effective treatment. Currently, the assessment of internal 
experiences, such as anxiety, depression, and loneliness, relies heavily on children's self-report. Whereas parents or teachers might be able to report easily on children's external behavior (e.g., temper tantrums, defying authority), they may have less insight into children's internal experiences (e.g., anxious thoughts, feelings of loneliness or depression). As such, clinical wisdom emphasizes the use of children's self-reports when assessing their internal experiences.

However, there is reason to question whether relying solely on self-reports of internal experiences is appropriate for all children with ADHD. Some children with ADHD display a "positive bias," meaning that they provide ratings of their abilities and experiences in different areas of their lives (e.g., social, academic, athletic) that are more positive relative to parent ratings, teacher ratings, or other objective measures, such as a math test. Because children with ADHD are more likely than their typically-developing peers to have a positive bias, ${ }^{4}$ it is important to consider how this bias may impact children's self-reports in other areas of their lives, such as co-occurring anxiety, depression, or loneliness.

In order to learn more about whether positive bias might play a role in children's ratings of their internalizing experiences, we examined data from two different groups of school-aged children (ages 7-12). The first group had 199 boys with ADHD and 84 typically-developing boys. The second group had 178 boys and girls with ADHD and 86 typically-developing children. Based on research linking children's social competence and their self-reports of internalizing problems, ${ }^{5,6,7}$ we focused specifically on children's social self-perceptual biases (i.e., the difference between child self-report and teacher report of social competence in areas related to making friends, spending time with peers, etc.) and examined whether this particular type of bias masked children's self-reports of loneliness, and depressive and anxious symptoms.

Results indicated that ADHD was more strongly linked with internalizing problems (anxiety, depression, loneliness) when children's social self-perceptual biases were considered as compared to when they were not. These findings provide initial support for the view that more positive social self-perceptual biases might interfere with the accurate assessment of loneliness, and depressive and anxious symptoms among some children with ADHD. In addition, our study showed that other types of self-perceptual biases (e.g., academic, behavioral) also masked the severity of internalizing problems among some children with ADHD. 
Findings from this study challenge the view that mental health clinicians should rely heavily on children's selfreport of internalizing problems. Specifically, our results suggest that children diagnosed with ADHD who also have a positive self-perceptual bias might underreport their experience of anxiety, depression, and feelings of loneliness. More research is needed to develop specific guidelines for clinicians to use in their daily clinical practice. For example, because self-perceptual biases range in size, we need a better understanding of the extent to which internalizing experiences are masked at different values of bias. This future work would allow clinicians to administer a self-perceptual bias measure and know at what point they need to make adjustments to assessment procedures.

Overall, results from this study highlight the need for mental health clinicians to consider how selfperceptual biases may impact the self-reports of some children with ADHD, particularly as this bias relates to the assessment of co-occurring internalizing mental health problems.

\section{Key Points}

- Children with ADHD often present with other mental health symptoms (anxiety, depression, loneliness). It is important to assess these co-existing problems to provide the needed treatment for these children.

- A positive self-perceptual bias (e.g., overestimation of own abilities) present among some children diagnosed with ADHD may hinder the accurate assessment of other mental health problems. Specifically, children with ADHD and a positive self-perceptual bias might provide underestimations of symptoms of anxiety, depression, and loneliness.

- Mental health clinicians may need to consider other sources of information (parent, teacher, coaches) when assessing internalizing problems among children with ADHD.

\section{References}

${ }^{1}$ Polanczyk, G., Rohde, L. A. Epidemiology of attention-deficit/hyperactivity disorder across the lifespan. Curr Opin Psychiatry. 2007;20(4):386-392.

2 Jarrett, M. A., Ollendick, T. H. A conceptual review of the comorbidity of attention-deficit/hyperactivity disorder and anxiety: Implications for future research and practice. Clin Psychol Rev. 2008;28(7):1266-1280.

3 Brown, R. T., Freeman, W. S., Perrin, J. M., et al. Prevalence and assessment of Attention-Deficit/ Hyperactivity Disorder in primary care settings. Pediatrics. 2001;107(3).

${ }^{4}$ Hoza, B., Gerdes, A. C., Hinshaw, S. P., et al. Self-perceptions of competence in children with ADHD and comparison children. J Consult Clin Psychol. 2004;72(3):382 - 391.

${ }^{5}$ Bornstein, M. H., Hahn, C.-S., Haynes, O. M. Social competence, externalizing, and internalizing behavioral adjustment from early childhood through early adolescence: developmental cascades. Dev Psychopathol. 2010;22(4):717-735.

${ }^{6}$ Cassidy, J., Asher, S. R. Loneliness and peer relations in young children. Child Dev. 1992;63(2):350-365.

7 Ladd, G. W., Troop-Gordon, W. The role of chronic peer difficulties in the development of children's psychological adjustment problems. Child Dev. 2003;74(5):1344-1367. 